\title{
Description of High-Energy pp Collisions Using Tsallis Thermodynamics: Transverse Momentum and Rapidity Distributions
}

\author{
L. Marques ${ }^{1}$, J. Cleymans ${ }^{2}$ and A. Deppman ${ }^{1}$ \\ ${ }^{1}$ Instituto de Física, Universidade de São Paulo - IFUSP, Rua do Matão, Travessa R 187, 05508-900 \\ São Paulo-SP, Brazil \\ ${ }^{2}$ UCT-CERN Research Centre and Department of Physics, University of Cape Town, Rondebosch \\ 7701, South Africa
}

\begin{abstract}
A systematic analysis of transverse momentum and rapidity distributions measured in high-energy proton - proton (pp) collisions for energies ranging from $53 \mathrm{GeV}$ to $7 \mathrm{TeV}$ using Tsallis thermodynamics is presented. The excellent description of all transverse momentum spectra obtained in earlier analyses is confirmed and extended. All energies can be described by a single Tsallis temperature of $68 \pm 5 \mathrm{MeV}$ at all beam energies and particle types investigated (43 in total). The value of the entropic index, $\mathrm{q}$, shows a wider spread but is always close to $\mathrm{q} \approx 1.146$. These values are then used to describe the rapidity distributions using a superposition of two Tsallis fireballs along the rapidity axis.

It is concluded that the hadronic system created in high-energy $\mathrm{p}$ - $\mathrm{p}$ collisions between $53 \mathrm{GeV}$ and $7 \mathrm{TeV}$ can be seen as obeying Tsallis thermodynamics.
\end{abstract}

\section{Introduction}

Recently a power-law function based on the Tsallis distribution has been used extensively in fits of the $p_{T}$ distributions measured in high-energy collisions [1 5$]$. The relationship with an approach based on Tsallis thermodynamics [6-8] (and not simply the distribution associated with it) has been clarified in several papers $9 \sqrt{12}$, in particular, the exponent appearing in the probability distribution used in [1 5$]$ is associated with the entropic index, $q$ forming the basis of Tsallis thermodynamics. A further step was made in [12] to generalize Hagedorn's 
theory [13, 14 to non-extensive thermodynamics that predicts a limiting temperature $(T)$ and a characteristic entropic index for hadronic systems 12$]$.

In this paper a systematic analysis of transverse momentum and rapidity distributions measured in high-energy proton - proton (pp) collisions for energies ranging from $53 \mathrm{GeV}$ to 7 $\mathrm{TeV}$ using Tsallis thermodynamics is presented. The excellent description of all transverse momentum spectra obtained in earlier analyses is confirmed and extended. The present analysis of $p_{T}$-distributions confirms previous results $10,11,15,16$ giving constant temperature and constant entropic index when the non-extensive distribution for systematic analysis of experimental data. A recent analysis [17, 18 obtained a higher temperature but it can be shown that this is due to not using a summation over all charged particles produced but simply fitting a single formula, doing so makes it consistent with the temperature $T$ used in this paper. In this paper these values are used to analyse rapidity distributions. In this case we use a model to describe the fireball rapidity distribution along the rapidity axis and show that a consistent pattern emerges which allow us to make extrapolations for the upcoming pp collision at the upgraded LHC.

In what follows we present a new analysis of $p_{T}$-distribution extending those already published, and provide more precise values for $T$ and $q$. Then we present a model based on the theory for describing rapidity distribution and perform a systematic analysis of experimental data on rapidity distribution of charged hadrons is performed. Finally the results of the analysis are used for predicting $p_{T}$ and rapidity distributions for the next LHC phase at $13 \mathrm{TeV}$.

\section{Transverse Momentum Distributions}

The power-law distribution based on Tsallis thermodynamics is given, in terms of transverse momentum and rapidity, by 10

$$
\frac{d^{2} N}{p_{T} d p_{T} d y}=g V \frac{m_{T} \cosh y}{(2 \pi)^{2}}\left[1+(q-1) \frac{m_{T} \cosh y-\mu}{T}\right]^{-\frac{q}{q-1}}
$$

where $\mu$ is the chemical potential, $m_{T}=\sqrt{p_{T}^{2}+m_{0}^{2}}, V$ is the volume, $g$ is the degeneracy factor and $q$ is the entropic factor, which measures the non-additivity of the entropy. Boltzmann statistics is recovered with $q=1$. This equation can be obtained also from a non-extensive version of the perfect gas partition function $[19$.

It has been shown that at central rapidity $y=0$ one can easily obtain the transverse 
momentum distribution in terms of the central rapidity density, $\left.\frac{d N}{d y}\right|_{y=0}$, as 20

$\left.\frac{d^{2} N}{d p_{T} d y}\right|_{y=0}=\left.\frac{p_{T} m_{T}}{T} \frac{d N}{d y}\right|_{y=0} \frac{(2-q)(3-2 q)}{(2-q) m_{0}^{2}+2 m_{0} T+2 T^{2}}\left[1+(q-1) \frac{m_{0}}{T}\right]^{\frac{1}{q-1}}\left[1+(q-1) \frac{m_{T}}{T}\right]^{-\frac{q}{q-1}}$

The above equation will be used for a systematic analysis of $p_{T^{-}}$distribution for pp collisions for a large set of experiments, as listed in Table 1. A subset of those experiments was already analysed in Ref. [21]. Since all experiments report results for a narrow range of rapidity around the central region the approximation used is appropriate. The only free parameters to be adjusted to the experimental data are $q$ and $T$. According to the thermodynamical theory in Ref. [12], if both the self-consistent principle from Hagedorn [13] and the Tsallis statistics [6 8] can be applied in high-energy physics then both $T$ and $q$ must be independent of particle type.

Table 1:

\begin{tabular}{|c|c|c|c|c|c|c|c|c|c|}
\hline Index & Particle & $\sqrt{s}(T e V)$ & Exp. & Ref. & Index & Particle & $\sqrt{s}(T e V)$ & Exp. & Ref. \\
\hline 1 & $\pi^{+}$ & 0.2 & PHENIX & 22 & 22 & $P^{-}$ & 0.9 & CMS & 24 \\
\hline 2 & $\pi^{-}$ & 0.2 & PHENIX & 22 & 23 & $\Lambda$ & 0.9 & CMS & 5 \\
\hline 3 & $K^{+}$ & 0.2 & PHENIX & 22 & 24 & $\Xi^{-}$ & 0.9 & CMS & 5 \\
\hline 4 & $K^{-}$ & 0.2 & PHENIX & 22 & 25 & $\pi^{+}$ & 2.76 & CMS & 24 \\
\hline 5 & $P^{+}$ & 0.2 & PHENIX & 22 & 26 & $\pi^{-}$ & 2.76 & CMS & 24 \\
\hline 6 & $P^{-}$ & 0.2 & PHENIX & 22 & 27 & $K^{+}$ & 2.76 & CMS & 24 \\
\hline 7 & $P^{+}$ & 0.2 & PHENIX & 22 & 28 & $K^{-}$ & 2.76 & CMS & 24 \\
\hline 8 & $P^{-}$ & 0.2 & PHENIX & 22 & 29 & $P^{+}$ & 2.76 & CMS & {$[24$} \\
\hline 9 & $\pi^{0}$ & 0.9 & ALICE & 23 & 30 & $P^{-}$ & 2.76 & CMS & 24 \\
\hline 10 & $\pi^{+}$ & 0.9 & ALICE & 3 & 31 & $\pi^{0}$ & 7.0 & ALICE & 23 \\
\hline 11 & $\pi^{-}$ & 0.9 & ALICE & 3 & 32 & $\pi^{+}$ & 7.0 & CMS & 24 \\
\hline 12 & $\pi^{+}$ & 0.9 & CMS & 24 & 33 & $\pi^{-}$ & 7.0 & CMS & 24 \\
\hline 13 & $\pi^{-}$ & 0.9 & CMS & 24 & 34 & $K_{s}^{0}$ & 7.0 & CMS & 5 \\
\hline 14 & $K_{s}^{0}$ & 0.9 & CMS & 5 & 35 & $K^{+}$ & 7.0 & CMS & 24 \\
\hline 15 & $K^{+}$ & 0.9 & ALICE & 3 & 36 & $K^{-}$ & 7.0 & CMS & 24 \\
\hline 16 & $K^{-}$ & 0.9 & ALICE & 3 & 37 & $\eta$ & 7.0 & ALICE & 23 \\
\hline 17 & $K^{+}$ & 0.9 & CMS & 24 & 38 & $K^{*}$ & 7.0 & ALICE & 25 \\
\hline 18 & $K^{-}$ & 0.9 & CMS & 24 & 39 & $P^{+}$ & 7.0 & CMS & 24 \\
\hline 19 & $P^{+}$ & 0.9 & ALICE & 3 & 40 & $P^{-}$ & 7.0 & CMS & 24 \\
\hline 20 & $P^{-}$ & 0.9 & ALICE & 3 & 41 & $\phi$ & 7.0 & ALICE & 25 \\
\hline 21 & $P^{+}$ & 0.9 & CMS & 24 & 42 & $\Lambda$ & 7.0 & CMS & 5 \\
\hline
\end{tabular}




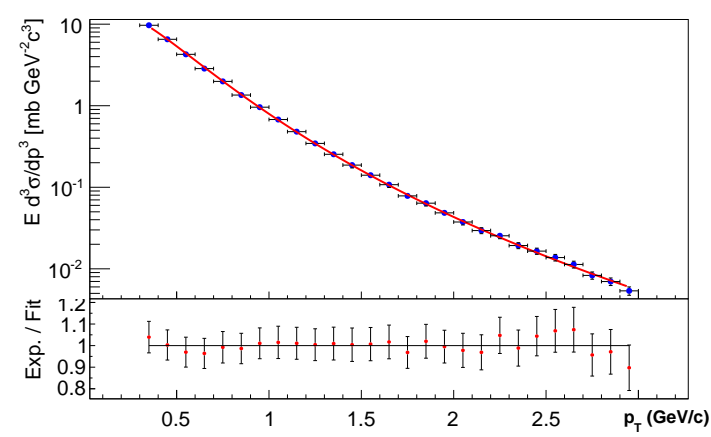

(a)

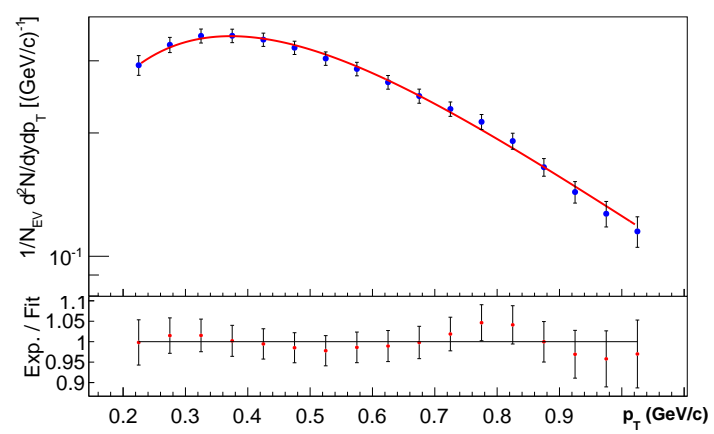

(c)

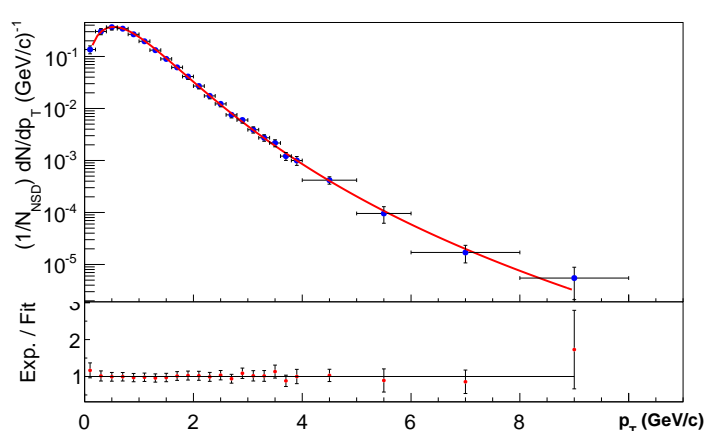

(b)

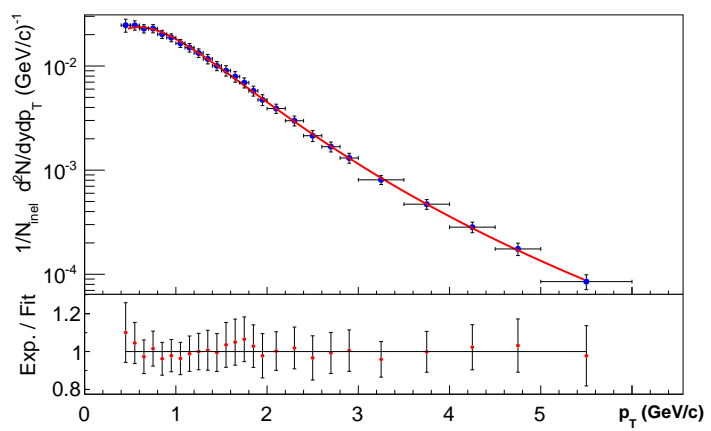

(d)

Figure 1: Fits to the $p_{T^{-}}$distribution given by Eq. 6 to experimental data. (a) $\pi^{+}, \sqrt{s}=0.2$ $\mathrm{TeV},[22]$ (b) $\Lambda, \sqrt{s}=0.9 \mathrm{TeV},[5]$ (c) $K^{-}, \sqrt{s}=2.76 \mathrm{TeV},[24]$ and (d) $\phi, \sqrt{s}=7.0 \mathrm{TeV},[25]$.

In Fig. 1 we show a typical result for the fitting of Eq. 2 to measured $p_{T}$-distribution. With the fittings for all experimental data used here we obtain the values for $T$ and $q$ that are plotted in Fig. 2, where we observe a distribution of both temperature and entropic index around a constant value.

The results obtained here are in agreement with previous analysis both qualitatively and quantitatively. Therefore we can determine the mean values from the analyses $10,15,16,21$, obtaining $q=1.146 \pm 0.004$ and $T=68 \pm 5 \mathrm{MeV}$. With these values all the thermodynamical aspects of the hot fireball are determined. 


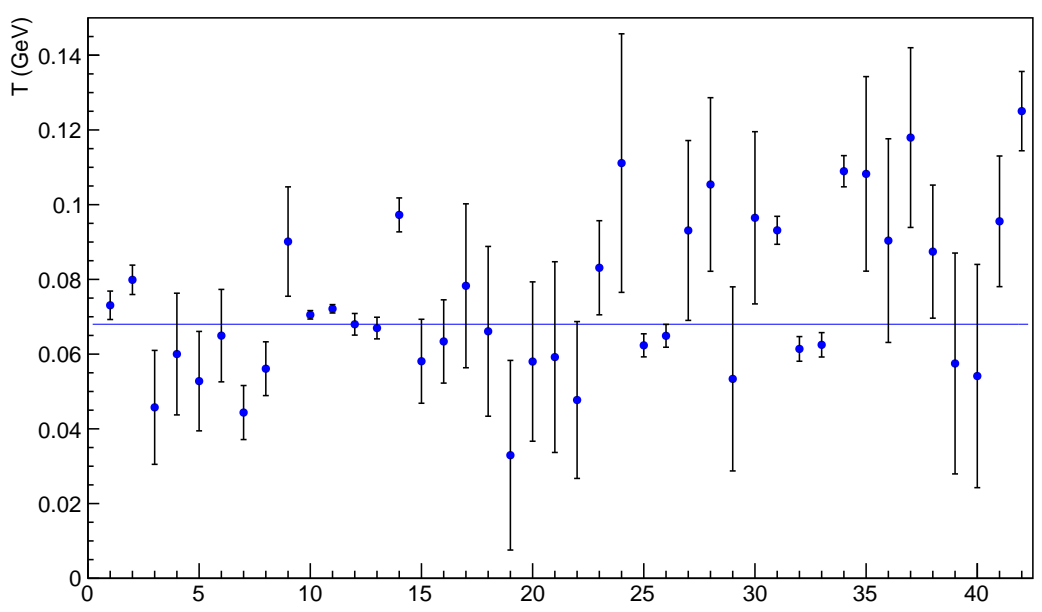

(a)

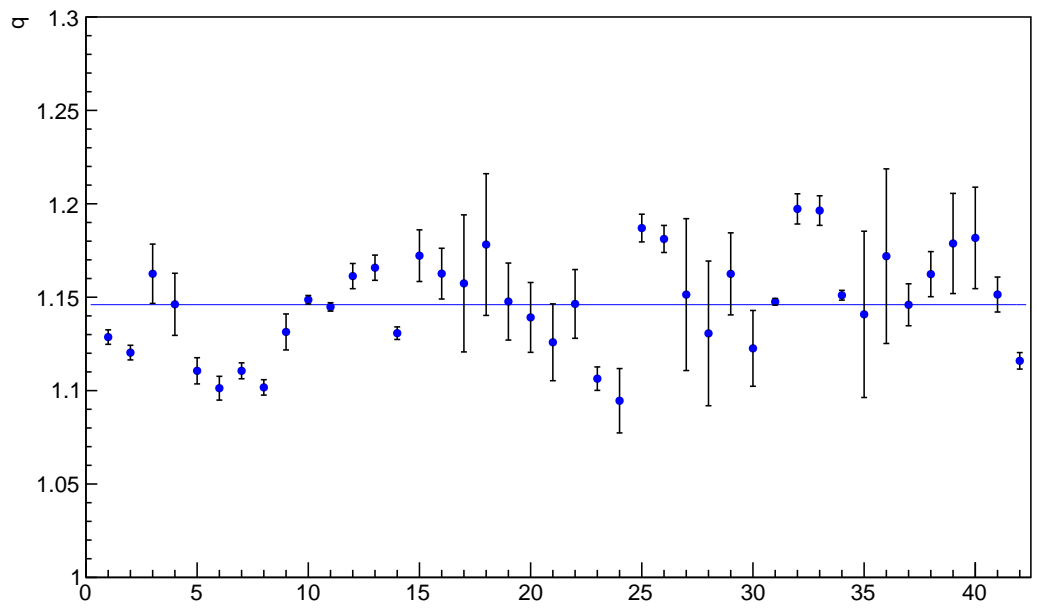

(b)

Figure 2: Results for the temperature, $T$ and for the entropic index, $q$, for all the sets of data analysed in this work. The numbering on the abscissa corresponds to the entries in Table 1. 


\section{Rapidity Distribution}

In the Bjorken model [26] a plateau in the central region of the rapidity distribution exists reflecting the invariance of the fireball under Lorentz transformation for boosts in the beam direction. Experimental results for high energy collisions, however, have shown that such a plateau does not exist, data showing two small peaks symmetrically located near the central rapidity region.

A systematic analysis performed in Ref. [27] shows that color-glass-condensate (CGC) approaches give a good description of the results at the central region but fail to reproduce the data at large values of $|y|$. Here we use a model for describing the rapidity distribution in pp collision using the non-extensive distribution. A similar method was already used in Refs [28, 29] in terms of Boltzmann-Gibbs statistics but the present analysis keeps some differences with respect to the previous one, as will be detailed below.

Suppose that the fireball is moving in the laboratory frame in the beam direction with rapidity $y_{f}$. According to Eq. 1 the yield of secondaries from the decay of this fireball is given by

$$
\frac{d^{2} N}{p_{T} d p_{T} d y}=g V \frac{m_{T} \cosh y^{\prime}}{(2 \pi)^{2}}\left[1+(q-1) \frac{m_{T} \cosh y^{\prime}-\mu}{T}\right]^{-\frac{q}{q-1}}
$$

where $y=y^{\prime}+y_{f}$.

From now on we assume $\mu=0$ since we restrict ourselves to high energy pp collisions, and adopt a model for the fireball longitudinal expansion which is similar, but not identical, to the Bjorken scenario [26]. In the present model the system the basic fireballs have rapidity distributed according to the function $\nu\left(y_{f}\right)$ so that

$$
\frac{d^{2} N}{p_{T} d p_{T} d y}=\frac{N}{A} \int_{-\infty}^{\infty} \nu\left(y_{f}\right) \times \frac{m_{T} \cosh \left(y-y_{f}\right)}{(2 \pi)^{2}}\left[1+(q-1) \frac{m_{T} \cosh \left(y-y_{f}\right)}{T}\right]^{-\frac{q}{q-1}} d y_{f}
$$

where $\nu\left(y_{f}\right) d y_{f}$ is the number of fireballs with rapidity between $y_{f}$ and $y_{f}+d y_{f}, N$ is the fully integrated particle multiplicity and $A$ is a normalization constant ensuring that

$$
\frac{1}{N} \int_{-\infty}^{\infty} \int_{0}^{\infty} \frac{d^{2} N}{d p_{T} d y} d p_{T} d y=1
$$

It is possible to integrate Eq. 4 over the transverse momentum $p_{T}$, resulting in:

$$
\begin{aligned}
\frac{1}{N} \frac{d N}{d y}= & \frac{1}{A} \int_{-\infty}^{\infty} \nu\left(y_{f}\right)\left[T^{3} \operatorname{sech}^{2}\left(y-y_{f}\right)\left(\frac{(q-1) \cosh \left(y-y_{f}\right)}{T}\right)^{\frac{2 q-3}{q-1}}\left(m_{0}(q-1)+T \operatorname{sech}\left(y-y_{f}\right)\right)\right] \\
& \times\left[\frac{\left(m_{0}+\frac{T \operatorname{sech}\left(y-y_{f}\right)}{q-1}\right)^{-\frac{q}{q-1}}\left(-m_{0}^{2}(q-2)+2 m_{0} T \operatorname{sech}\left(y-y_{f}\right)+2 T^{2} \operatorname{sech}^{2}\left(y-y_{f}\right)\right)}{4 \pi^{2}(q-2)(q-1)^{3}(2 q-3)}\right] d y_{f}
\end{aligned}
$$


The equation above gives the rapidity distribution of particles produced in pp collisions and is the basis for describing the available experimental results at different beam energies.

For completeness we still need the distribution function $\nu\left(y_{f}\right)$. Based on the shape of the experimental distributions we choose the following ansatz:

$$
\nu\left(y_{f}\right)=G_{q^{\prime}}\left(y_{0}, \sigma ; y_{f}\right)+G_{q^{\prime}}\left(-y_{0}, \sigma ; y_{f}\right)
$$

where

$$
G_{q}\left(y_{0}, \sigma ; y_{f}\right)=\frac{1}{\sqrt{2 \pi} \sigma} e_{q}\left(-\frac{\left(y_{f}-y_{0}\right)^{2}}{2 \sigma^{2}}\right)
$$

and $e_{q}(x)$ is the q-exponential function defined as

$$
e_{q}(x) \equiv[1-(q-1) x]^{-1 /(q-1)}
$$

In Eq. $8 y_{0}$ and $\sigma$ are respectively the peak position and the width of the q-Gaussian function and are considered here as free parameters to be adjusted in a systematic analysis of experimental data. Eq. 7 assumes that the main final state in the collision is composed of two fireballs moving in the beam direction with opposite rapidities $y_{f}$, each of them being composed by fireballs with rapidity-gaps with respect to the corresponding cluster that are distributed according to a q-Gaussian function.

Notice that $q^{\prime}$ in Eq. 7 does not need to be equal to $q$ and in the present work we perform the analysis of experimental data with two different assumptions: $q^{\prime}=q$ and $q^{\prime}=1$, the last assumption corresponding to a Gaussian distribution of the rapidity-gap of the fireballs in each of the moving cluster. In addition, we assume that the fireballs are described by the nonextensive self-consistent thermodynamics with the limiting temperature $T$ and the characteristic entropic index, $q$, that were found in the analysis of $p_{T}$-distributions and hadronic spectrum, as described above. The values adopted here are $T=68 \mathrm{MeV}$ and $q=1.146$. For simplicity we will adopt $m_{0}=139.59 \mathrm{MeV}$, since most of the charged hadrons produced at high energy collisions are pions. The set of experimental data used for the rapidity analysis is shown in Table 2 .

The main difference between the analysis performed in the present work and that performed in Refs. 28, 29] is, of course, the use of Tsallis statistics in the thermodynamical description of the fireball however other differences must be noticed. While in [28] both temperature and chemical potential could depend on the fireball rapidity, here we adopt constant temperature at the limiting value predicted theoretically and null chemical potential regardless of the fireball rapidity. This approach is justified by the fact that our analysis focuses on charged hadrons distributions produced at very high beam energies $(>500 \mathrm{GeV})$, which are dominated by pion production. This assumption can be improved with the availability of rapidity distributions for identified hadrons 28 30]. 
Table 2:

\begin{tabular}{|c|c|c|c|}
\hline$\sqrt{s}(G e V)$ & Exp. & Range & Ref. \\
\hline 53 & UA5 & $|y| \leq 3.1$ & 31 \\
\hline 200 & UA5 & $|y| \leq 4.6$ & 31 \\
\hline 200 & PHOBOS & $|y| \leq 5.3$ & 32 \\
\hline 410 & PHOBOS & $|y| \leq 5.3$ & 32 \\
\hline 546 & UA5 & $|y| \leq 4.8$ & 31 \\
\hline 630 & UA5 & $|y| \leq 5.5$ & 33 \\
\hline 900 & UA5 & $|y| \leq 4.6$ & 31 \\
\hline 900 & CMS & $|y| \leq 2.5$ & 34 \\
\hline 1800 & $\mathrm{CDF}$ & $|y| \leq 3.5$ & 35 \\
\hline 2360 & CMS & $|y| \leq 2.5$ & 34 \\
\hline 7000 & CMS & $|y| \leq 2.5$ & 36 \\
\hline
\end{tabular}

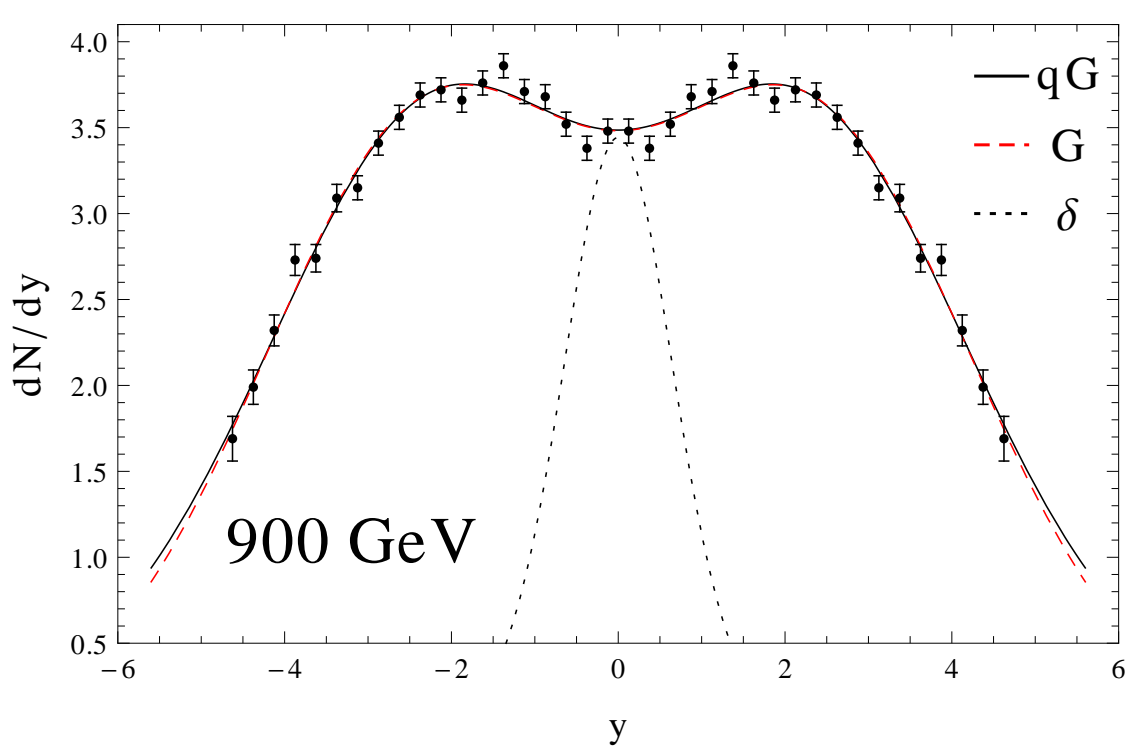

Figure 3: Fit using Eq. 6 to experimental data for rapidity distribution from pp collision at 900 GeV from the UA5 collaboration [31]. Data for $y<0$ are obtained symmetrically from $y>0$. The full line represents the best fit using the q-Gaussian function $\left(q^{\prime}=q\right)$ and the dashed line corresponds to the best fit using the Gaussian function $\left(q^{\prime}=1\right)$ in Eq. 7. The dotted line shows the results when a delta function is used for the fireball rapidity distribution, i.e., $\nu\left(y_{f}\right)=\delta\left(y_{f}\right)$.

In Fig. 4 we show the results of fits to the rapidity distributions of particles produced in pp collisions from several experiments with collision energies ranging from $50 \mathrm{GeV}$ up to $7 \mathrm{TeV}$. The set of experimental data used in the complete analysis is shown in Table 2. We observe a good fit of formula 6 to the experimental data in the whole range of rapidity available, which covers almost the entire rapidity region. These results and the others presented in Appendix A 
shows that a consistent description of the experimental data can be obtained with the model described above. Notice that the only free parameters in the fitting procedure are $\sigma$ and $y_{0}$ appearing in Eq. 7. From the analysis performed on all the sets of experimental data in Table 2 we get the values for those parameters that are presented in Fig. 3 .

For both quantities we observe a sharp increase up to collision energies around $500 \mathrm{GeV}$. The uncertainties above this energy are also larger due to the fact that the range of rapidity available is smaller. The striking feature of these results is the fact that both the peak position $y_{0}$ and the width $\sigma$ of the q-Gaussian remain within the same range i.e. are approximately constant for collision energies from $500 \mathrm{GeV}$ to $7 \mathrm{TeV}$, while the beam rapidity varies by a factor 2 in this range of collision energies. These results allow us to conclude that after the two original protons collide two clusters of fireballs are formed and they move parallel to the beam direction with opposite rapidities given by $\left|y_{0}\right|=2.3$. Each cluster is formed by fireballs that move with respect to the center of each cluster in the beam direction with rapidity distribution that is described by a q-Gaussian function with width $\sigma \approx 1.8$. We will assume in the rest of this paper that the values of $y_{0}$ and $\sigma$ will not change drastically for collision energies up the $14 \mathrm{TeV}$. Of course, it is expected that the position of $y_{0}$ will increase at much higher beam energies.

Below $\approx 550 \mathrm{GeV}$ the scaling observed above disappears, as can be seen in Fig. 4. It is possible that at these collision energies the temperature achieved by the system is significantly lower than the limiting temperature, $T$, hence more studies on the direction of verifying the role played e.g. by the chemical potential at low energies must be carried out, as performed in 28 30].

When we use the Gaussian function instead of the q-Gaussian for the rapidity distribution of the fireballs, i.e.,

$$
\nu\left(y_{f}\right)=G\left(y_{0}, \sigma ; y_{f}\right)+G\left(-y_{0}, \sigma ; y_{f}\right)
$$

with $G\left(y_{0}, \sigma ; y_{f}\right)$ being the Gaussian function with peak position at $y_{0}$ and width $\sigma$, the results of the fitting procedure Eq. 6 to the experimental data are very similar to those obtained with the q-Gaussian function, as shown in Fig. 3 with dashed line. The best fit values for the parameters $y_{0}$ and $\sigma$ are also practically the same and they are presented in Fig. 4 with open symbols.

\section{Energy Dependence of Multiplicity}

Once the parameters $T, q, y_{0}$ and $\sigma$ are determined, if we know the function $N(\sqrt{s})$ giving the multiplicity as a function of energy we can determine the $p_{T}$ and rapidity-distributions 


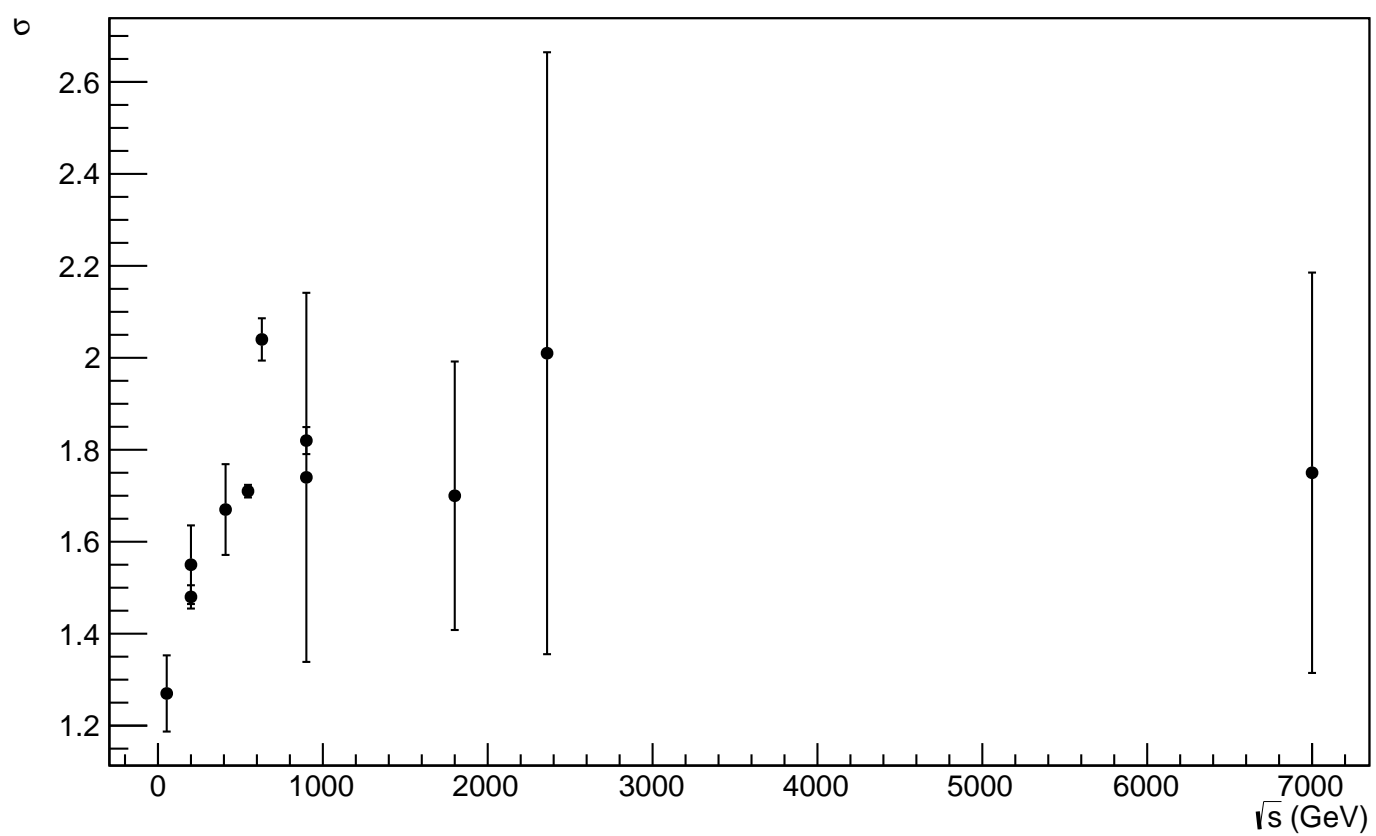

(a)

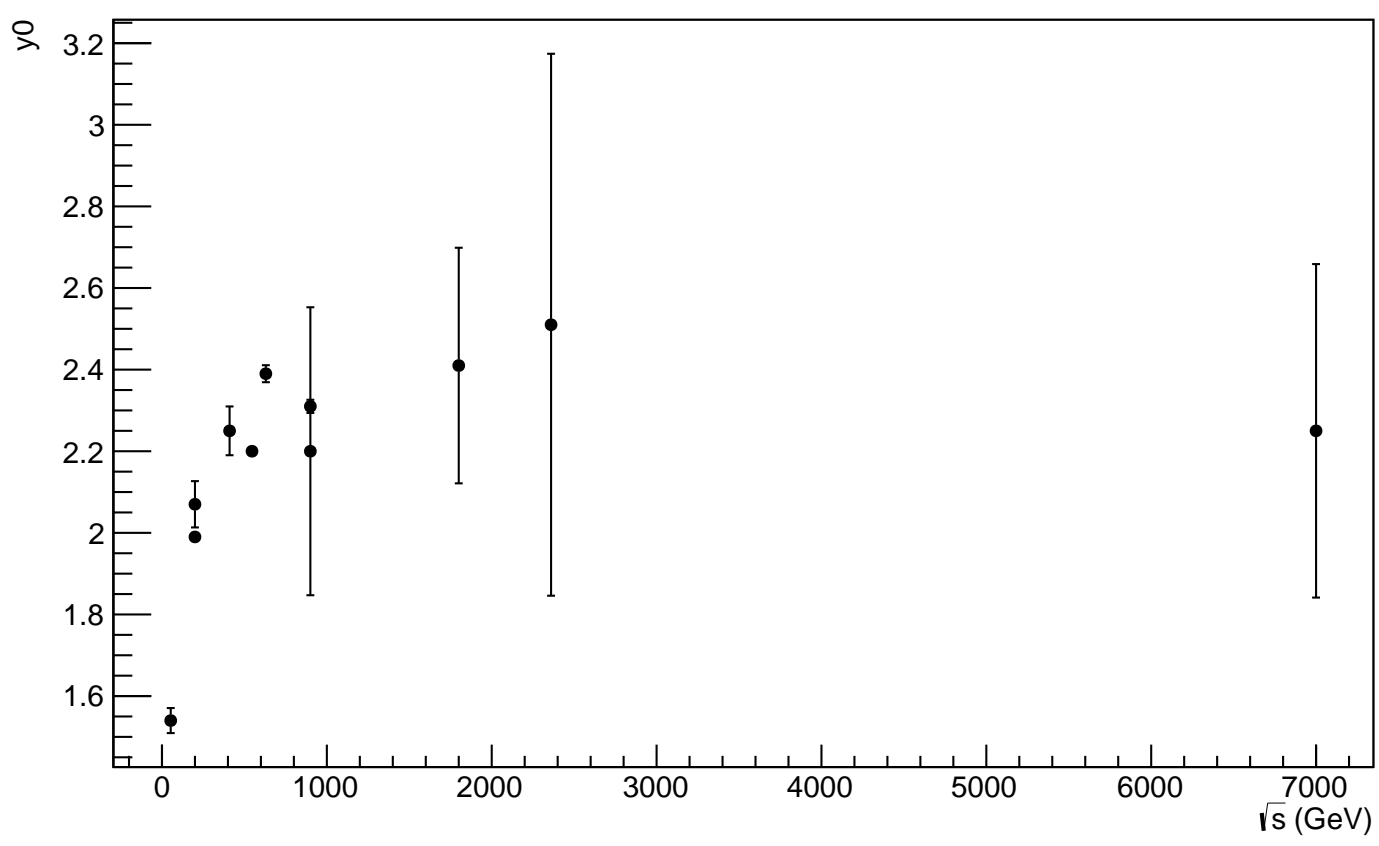

(b)

Figure 4: Values for the parameters $\sigma$ and $y_{0}$ in Eq. 6 as a function of the collision energy.

of secondaries produced in pp collisions at any collision energy sufficiently high to allow the assumption that those parameters are independent of beam energy and particle properties. 
Therefore now we investigate how the function $N(\sqrt{s})$ behaves.

The energy carried out by all particles with mass $m$ is determined by

$$
E(m)=\frac{g V}{(2 \pi)^{2}} \rho(m) \int_{-\infty}^{\infty} \int_{0}^{\infty} \varepsilon\left(p_{T}, y\right) \frac{d^{2} N}{d p_{T} d y} d p_{T} d y
$$

where

$$
\varepsilon\left(p_{T}, y\right)=m_{T} \cosh (y)
$$

The total energy carried by baryons is

$$
E=\int_{0}^{\infty} \rho(m) E(m) d m
$$

where $\rho(m)$ is the baryon mass spectrum. The function $\rho(m)$ was determined by the nonextensive self-consistent theory [12] and is given by

$$
\rho(m)=\frac{\gamma}{m^{5 / 2}}\left[1+\frac{(q-1) m}{T}\right]^{\frac{1}{q-1}}
$$

In Ref. 21] it was shown that this formula reproduces correctly the observed hadronic mass spectrum up to a mass of approximately $2 \mathrm{GeV}$.

Substituting Eq. 4 in Eqs. 11 and 13 we obtain a relation between the total baryonic energy and the multiplicity that is given by

$$
E=N(E) \frac{1}{A} \int_{0}^{\infty} \rho(m) \int_{-\infty}^{\infty} \int_{0}^{\infty} \varepsilon\left(p_{T}, y\right) \frac{d^{2} N}{d p_{T} d y} d p_{T} d y d m
$$

Notice that for sufficiently high energy, when $T, q, \sigma$ and $y_{0}$ do not depend on the collision energy, the only term which can depend on the total energy is the multiplicity, $N(E)$. At the limiting temperature the calculations are difficult to be performed because of the singularity in the partition function corresponding to the phase transition. Therefore we use a sum over a subset of the particles produced in high energy collisions, so that

$$
N(E)=K E,
$$

where

$$
K=\left[\frac{1}{A} \sum_{i=1}^{n} \int_{-\infty}^{\infty} \int_{0}^{\infty} \varepsilon\left(p_{T}, y\right) \frac{d^{2} N}{d p_{T} d y}\left(m_{i}\right) d p_{T} d y\right]^{-1}
$$

where the sum is over all the particles indicated in Table $3, m_{i}$ is the particle mass, and

$$
\frac{d^{2} N}{d p_{T} d y}\left(m_{i}\right)
$$

is the double differential yield given by Eq. 6 when the mass $m_{i}$ is used. 
Table 3:

\begin{tabular}{cc}
\hline Particle & Mass $\left(\mathrm{GeV} / \mathrm{c}^{2}\right)$ \\
\hline$\pi^{ \pm}$ & 0.140 \\
$K^{ \pm}$ & 0.494 \\
$\rho$ & 0.770 \\
$K^{*}$ & 0.892 \\
$p$ & 0.938 \\
$\Sigma^{+}$ & 1.189 \\
$\Sigma^{-}$ & 1.197 \\
$\Delta$ & 1.232 \\
$\Xi^{-}$ & 1.321 \\
$\Sigma^{*}$ & 1.385 \\
$\Xi^{*}$ & 1.533 \\
$\Omega^{-}$ & 1.672 \\
$D^{ \pm}$ & 1.869 \\
$F^{ \pm}$ & 1.971 \\
$D^{*}$ & 2.010 \\
$\Lambda_{c}^{+}$ & 2.281 \\
$B^{ \pm}$ & 5.271 \\
\hline
\end{tabular}

With Eq. 17 and using all particles listed in Table 3 we get $K=0.009 \mathrm{GeV}^{-1}$. This value is above the best fitting value $K=0.004 \mathrm{GeV}^{-1}$, showing that the more massive particles not included in our calculation give a significant contribution to the total energy. In Fig. 5 we show the linear variation of multiplicity using the calculated value for $K$ as a dashed line.

In Fig. 5 we present the multiplicity $N$ obtained from the fittings of Eq. 6 to the rapidity distribution data using the q-Gaussian function for the fireball rapidity distribution (black symbols). We observe that after a fast increase for collision energies up to $\sim 500 \mathrm{GeV}$ the multiplicity increases more slower at higher energies. As already discussed, for low energies we cannot ensure that $T$ is constant and that the chemical potential is null, therefore we will not deepen our discussion for the results at those energies. Nonetheless we fitted the multiplicity data with two different fitting formulas: one linear for energies above $500 \mathrm{GeV}$ and the other logarithm for the full rapidity range. The results are plotted in Fig. 5.

We observe that the linear fit has a coefficient very different from $K$ calculated above. Indeed, the curve one would obtain with this coefficient is the dotted line also shown in that figure, which gives a consistent description of the multiplicity in the energy range from $500 \mathrm{GeV}$ up to $2500 \mathrm{GeV}$ but would not describe correctly the multiplicity at $7 \mathrm{TeV}$. This happens because the coefficient $K$ consider only the contribution from the hadrons listed in Table 3 , which results in an overestimation of the total energy carried by all hadrons according to Eq. 16. 


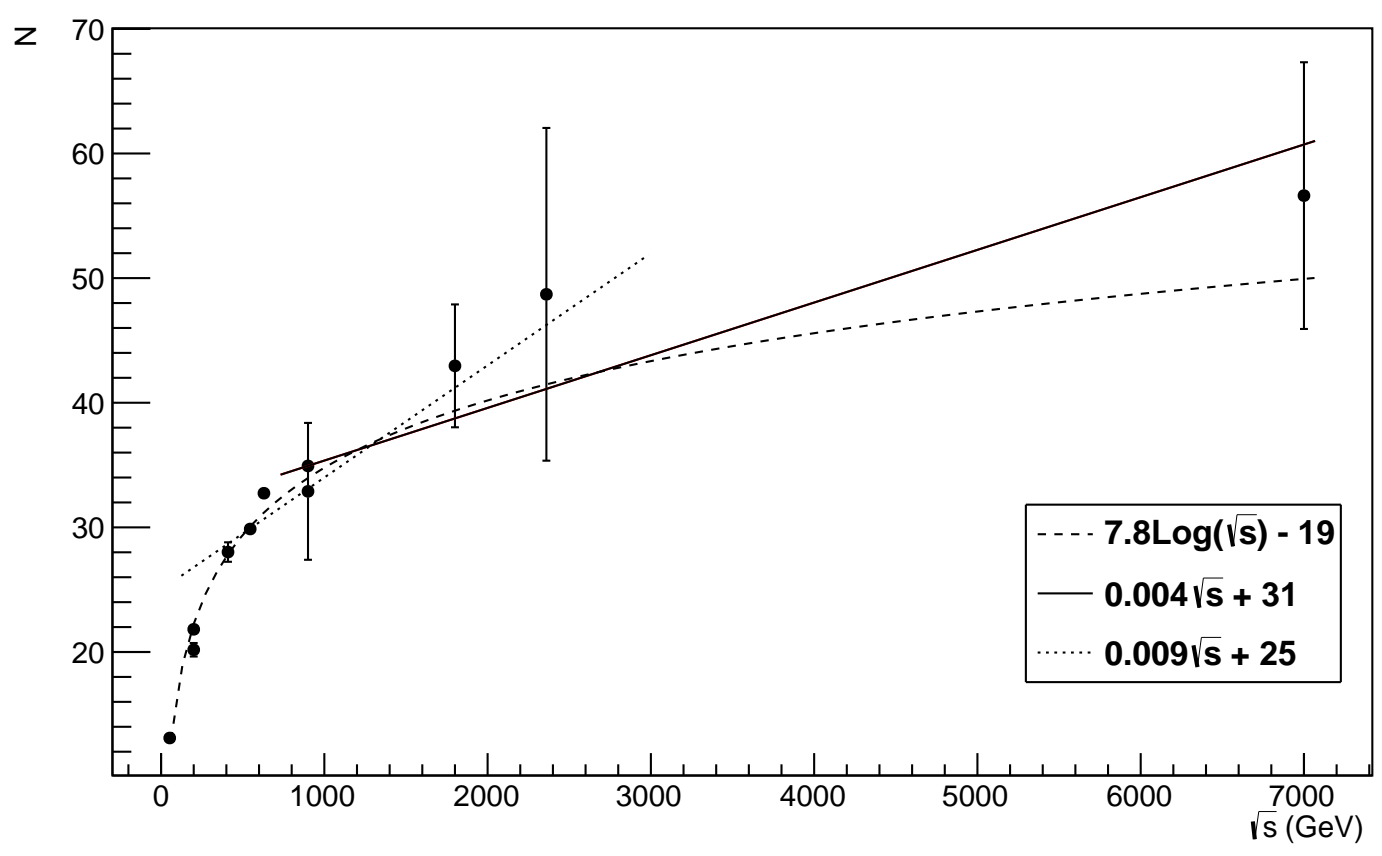

Figure 5: The particle multiplicity $N$ as a function of $\sqrt{s}$. The solid line is a linear fit to the data for $\sqrt{s}>800 \mathrm{GeV}$. The dashed line represents the logarithm function. The dotted line is the multiplicity as a function of energy when the coefficient $K$ is used.

The problem is that as the collision energy increases, small variations in the temperature, which is tending to the limiting temperature, will result in a sharp increase of the number of massive hadrons, as described by $\rho(m)$, and these particles contribute significantly to the total energy even if they have small contributions to the total multiplicity. Therefore the linear fit in Fig. 5 can be estimated as an upper limit to the multiplicity for energies above $7 \mathrm{TeV}$. The logarithm fit, on the other hand, could be considered as the lower limit, since it systematically underestimate the charged hadrons multiplicities for collision energies above $2 \mathrm{TeV}$.

As examples of application of the present model we now calculate the $p_{T^{-}}$and $y$-distributions for charged particles at collision energy $E=13 \mathrm{TeV}$, which is the expected energy at the LHC experiments.

The rapidity distribution is shown in Fig. 6 using the linear extrapolation (full line), which gives a total multiplicity $M=83$, and the logarithm extrapolation, which gives $M=55$. These results are obtained with q-Gaussian functions for $\nu\left(y_{f}\right)$ with $y_{0}=2.4$ and $\sigma=1.8$.

The estimates for $p_{T}$-distribution of charged particles are shown in Fig. 7. The lines indicate the lower and the upper limits of our theoretical calculations using Eq. 2 and considering the uncertainties on the parameters $q$ and $T$. The uncertainty in the multiplicity is not taken into account but we present the results obtained with both extrapolations for $N(E)$, namely, the 


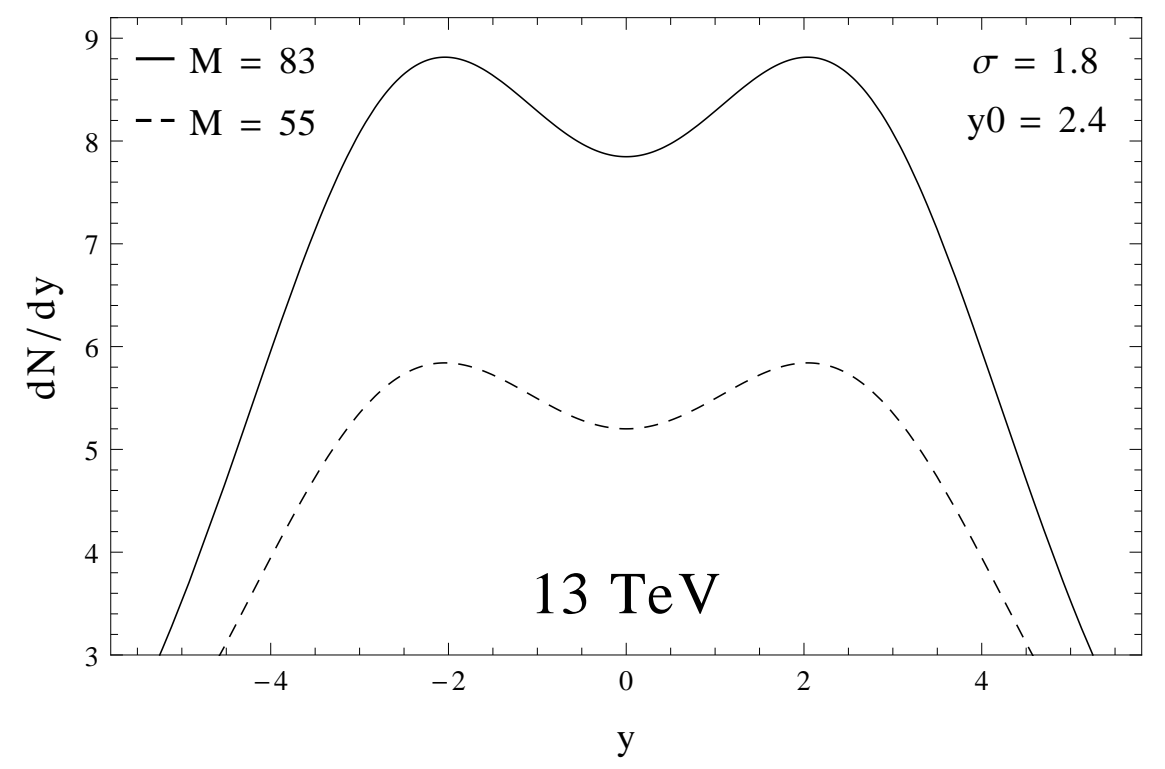

Figure 6: The charged hadrons rapidity-distribution predicted for $13 \mathrm{GeV}$ pp collisions. The full line corresponds to the multiplicity calculated through the linear extrapolation and the dashed line corresponds to the multiplicity obtained with the logarithm extrapolation, as given in Fig. 5 .

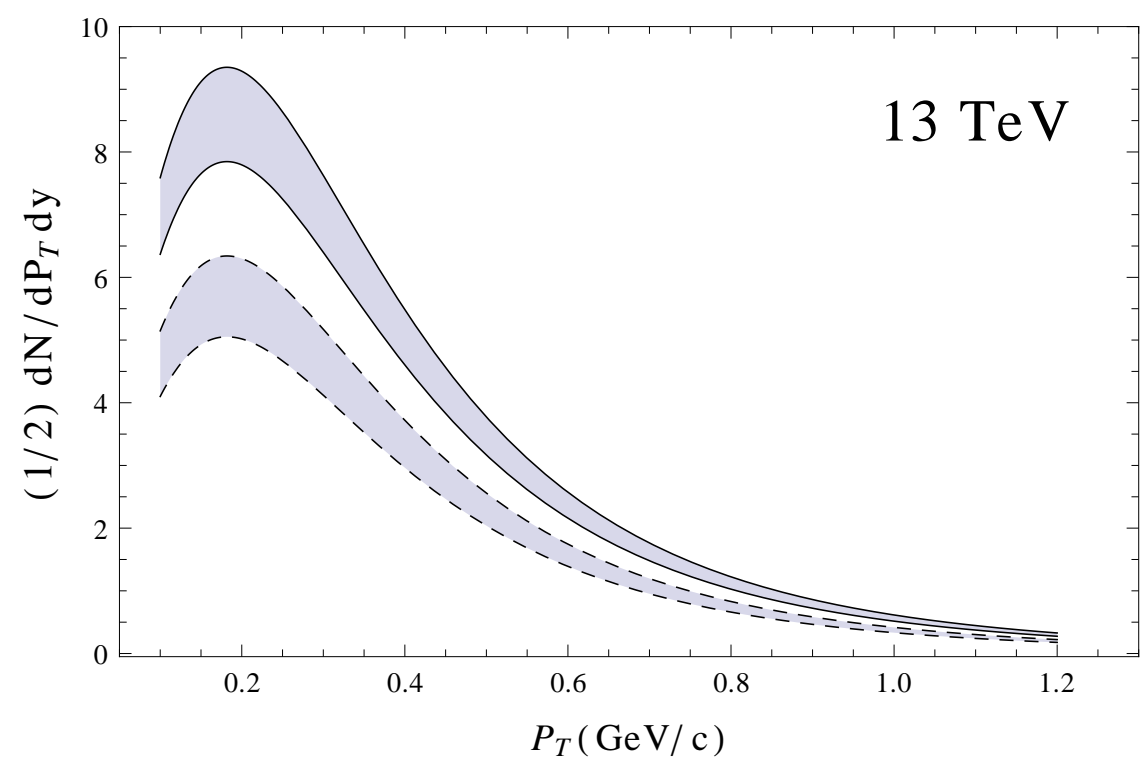

Figure 7: The charged hadrons $p_{T}$-distribution predicted for $13 \mathrm{GeV}$ pp collisions. The shaded regions show the expected distribution considering the uncertainties in the parameters $T$ and $q$. The upper region with full lines corresponds to the multiplicity calculated through the linear extrapolation and the bottom region with dashed lines corresponds to the multiplicity obtained with the logarithm extrapolation, as given in Fig. 5 . 
linear (full lines) and the logarithm (dashed lines). The shaded area between the lines show the region where we expect the data to be measured at LHC.

\section{Conclusions}

In this work we presented an analysis of the $p_{T}$ and rapidity distribution in pp collisions assuming that the final state can be described by two fireballs obeying Tsallis thermodynamics having a Tsallis temperature $T=68 \pm 5 \mathrm{MeV}$ and an entropic index $q=1.146 \pm 0.004$ independent of beam energy.

Our results on the transverse momentum distributions are in agreement with previous analysis and allows a complete characterisation of the thermodynamics of fireballs.

We developed a model to describe the rapidity distribution with non-extensive thermodynamics which is based on the assumption that the hot system formed after pp collisions can be represented as two clusters of fireballs moving in the beam direction with rapidities distributed according to a function $\nu\left(y_{f}\right)$. We use a sum of two q-Gaussian functions to describe $\nu\left(y_{f}\right)$. It results that for energies above $\sim 500 \mathrm{GeV}$ all rapidity distributions can be reproduced with constant peak-position and constant width for the q-Gaussian functions.

With these results we estimated the behaviour of the multiplicity as a function of the collision energy, allowing us to present estimates for the future LHC experiments at $13 \mathrm{TeV}$.

\section{Acknowledgment}

The authors are thankful to R. Martins and to S.F. Barros for their help with numerical calculations. This work received support from CNPq, under grant 305639/2010-2 (A.D.), and by FAPESP under grant 2014/21648-1 (L.M). 


\section{Appendix}

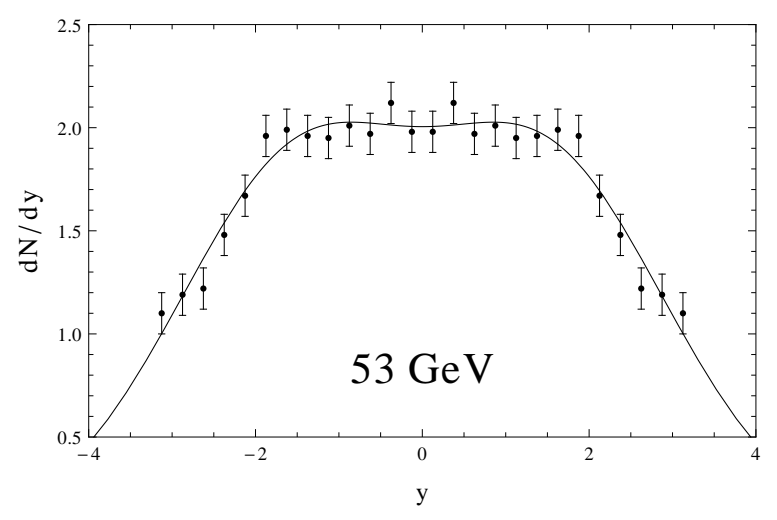

(a)

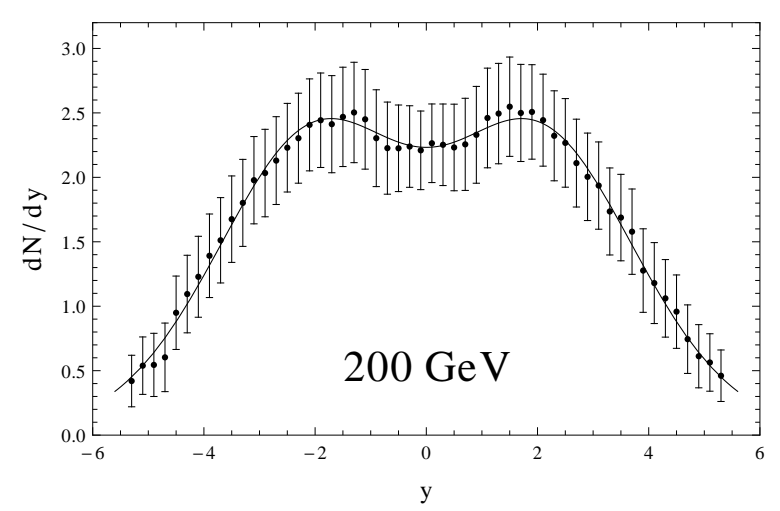

(c)

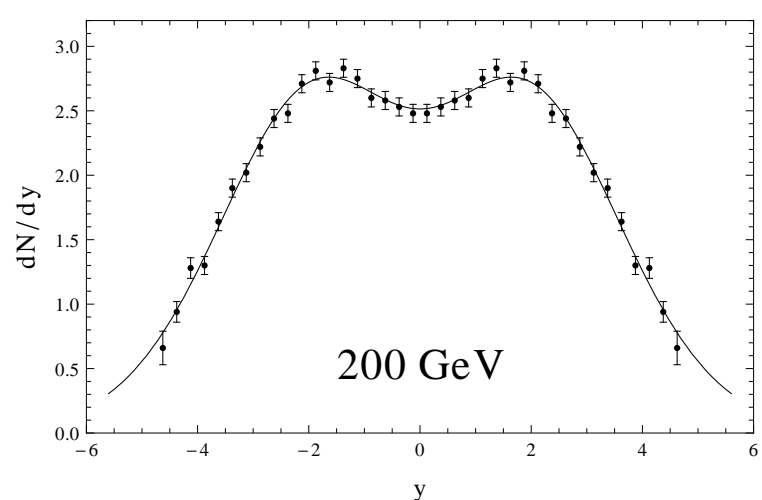

(b)

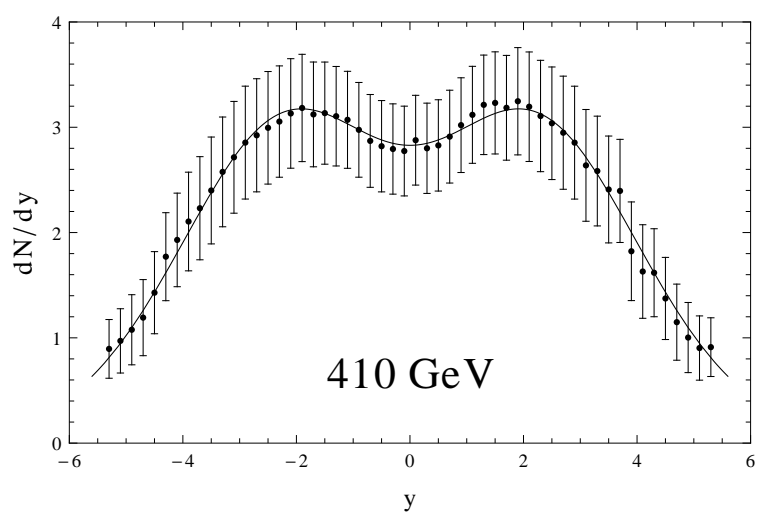

(d)

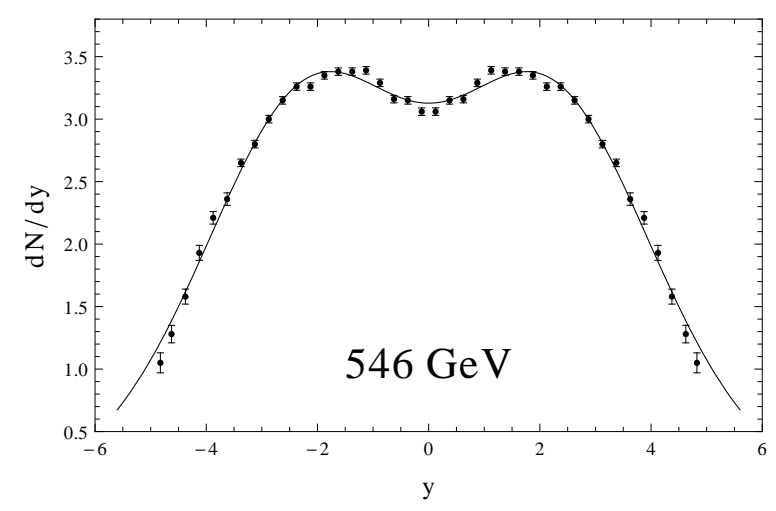

(e)

Figure 8: Fits to rapidity distributions measured in high-energy p-p collisions (see Table 2) with Eq. 6. The experimental data are from: (a) UA5 collaboration [31] (b) UA5 collaboration 31] (c) PHOBOS collaboration [32] (d) PHOBOS collaboration 32] (e) UA5 collaboration 31]. 


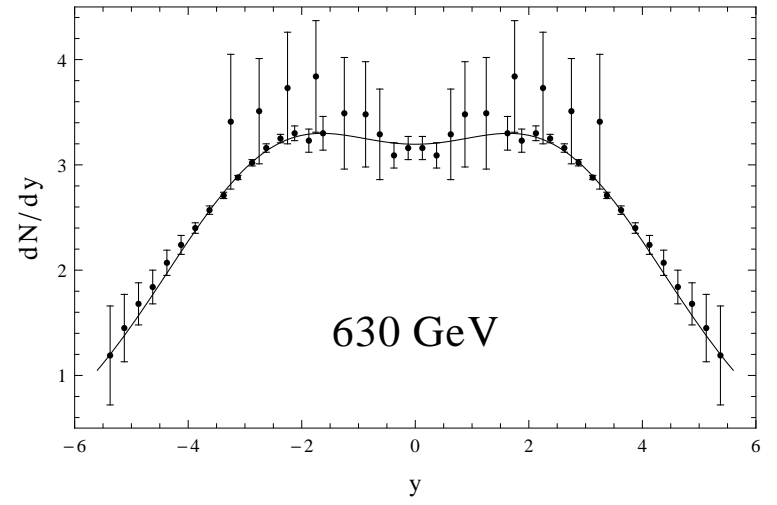

(a)

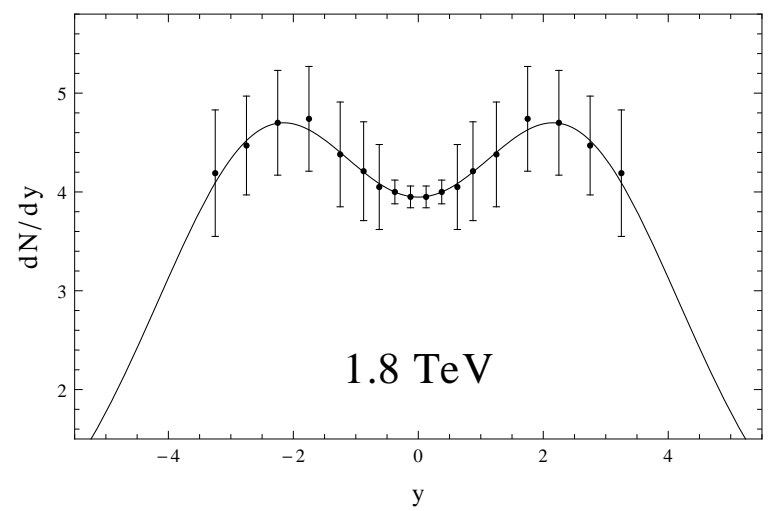

(c)

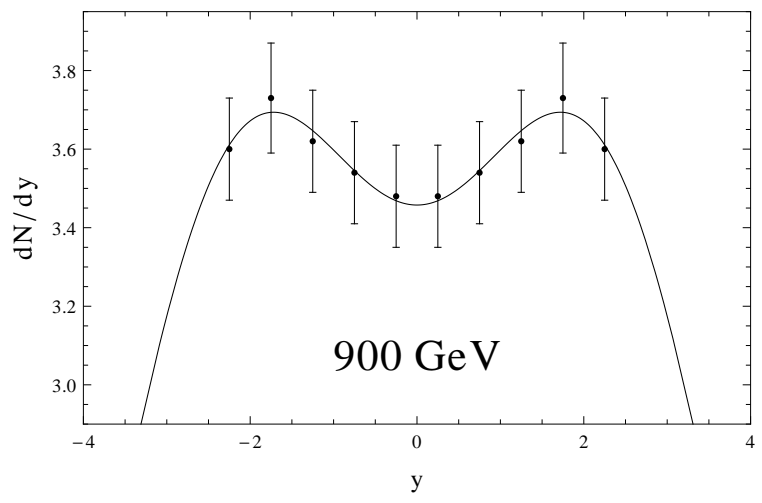

(b)

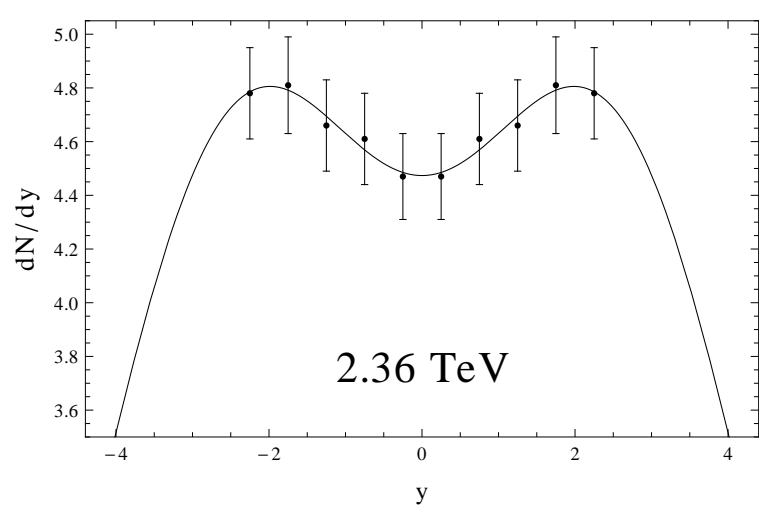

(d)

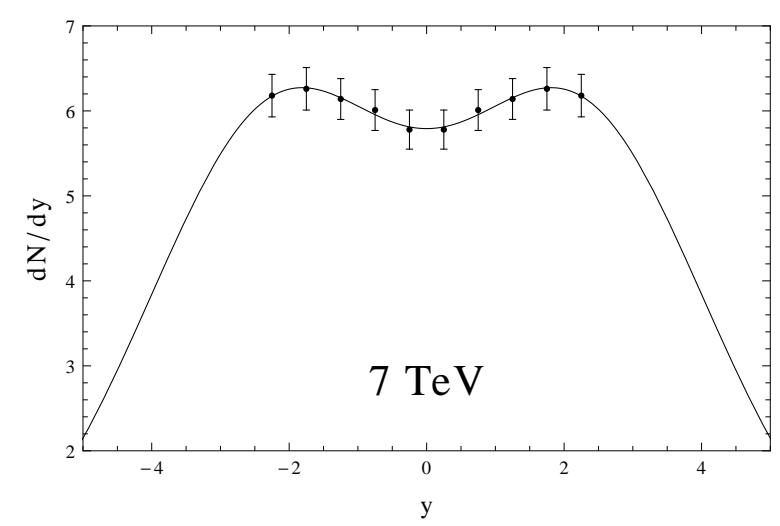

(e)

Figure 9: Series of fittings of experimental data on rapidity distribution (see Table 2) with Eq. 6. The experimental data are from: (a) UA5 collaboration [31] (b) CMS collaboration [34] (c) CDF collaboration [35] (d) CMS collaboration [34] (e) CMS collaboration 36] 


\section{References}

[1] B. I. Abelev et al. (STAR Collaboration), Phys. Rev. C 75, 064901 (2007).

[2] A. Adare et al. (PHENIX Collaboration), Phys. Rev. C 83, 052004, (2010); Phys. Rev. C 83, 064903 (2011).

[3] K. Aamodt, et al. (ALICE Collaboration), Eur. Phys. J. C 71 (2011) 1655.

[4] G. Aad, et al. (ATLAS Collaboration), New J. Phys. 13 (2011) 053033.

[5] V. Khachatryan, et al. (CMS Collaboration), JHEP 05 (2011) 064.

[6] C. Tsallis, J. Stat. Phys. 52 (1988) 479.

[7] C. Tsallis, Braz. J. Phys. 29 (1999) 1.

[8] T.S. Biro, "Is There a Temperature?" Fundamental Theories of Physics, Vol 171 (2011), Springer Verlag.

[9] C. Beck, Physica A 286 (2000) 164.

[10] J. Cleymans and D. Worku, J. Phys. G: Nucl. Part. Phys. 39 (2012) 025006.

[11] M.D. Azmi and J. Cleymans, J. Phys. G: Nucl. Part. Phys. 41 (2014) 065001.

[12] A. Deppman, Physica A 391 (2012) 6380.

[13] R. Hagedorn, Suppl. Nuovo Cimento 3 (1965) 147.

[14] R. Hagedorn, Ref.TH.3684-CERN, September (1983).

[15] I. Sena and A. Deppman, Eur. Phys. J. A 49 (2013) 17.

[16] I. Sena and A. Deppman, AIP Conf. Proc. 1520, 172 (2013).

[17] C.-Y. Wong and G. Wilk, Acta Phys. Polon. B 43, 2047 (2012).

[18] C.-Y. Wong and G. Wilk, Phys. Rev. D 87, 114007 (2013).

[19] E. Megías, D.P. Menezes and A. Deppman, Physica A 421 (2015) 15-24.

[20] M. Rybczyński, Z. Wlodarczyk, Eur. Phys. J. C 74 (2014) 2785.

[21] L. Marques, E. Andrade-II and A. Deppman, Phys. Rev. D 87 (2013) 114022. 
[22] A. Adare, et al. Phys. Rev. C 83 (2011) 064903.

[23] B. Abelev, et al. Phys. Lett. B 717 (2012) 162.

[24] S. Chatrchyan, et al. Eur. Phys. J. C 72 (2012) 2164.

[25] B. Abelev et al. (ALICE Collaboration), Eur. Phys. J. C 72 (2012) 2183.

[26] J.D. Bjorken, Phys. Rev. D 27 (1983) 140.

[27] A. Kumar, P.K. Srivastava, B.K. Singh and C.P. Singh, Adv. High En. Phys. 2013 (2013) 352180 .

[28] F. Becattini and J. Cleymans, J. Phys. G: Nucl. Part. Phys. 34 (2007) S959-S963.

[29] J. Cleymans, J. Phys. G: Nucl. Part. Phys. 35 (2008) 044017.

[30] L.A. Stiles and M. Murray,nucl-ex/0601039.

[31] G. J. Alner, Z. Phys. C - Particles and Fields 33 (1986) 1.

[32] B. Alver, et al. Phys. Rev. C 83 (2011) 024913.

[33] R. Harr, et al. Phys. Lett. B 401 (1997) 176.

[34] V. Khachatryan, et al. J. High Energy Phys. 2 (2010) 041.

[35] F. Abe, et al. Phys. Rev. D (R) 41 (1990) 2330.

[36] V. Khachatryan, et al. Phys. Rev. Lett. 105 (2010) 022002. 